\title{
The q-Coulomb problem in configuration space
}

A. C. Cadavid, and R. J. Finkelstein

Citation: Journal of Mathematical Physics 37, 3675 (1996); doi: 10.1063/1.531594

View online: http://dx.doi.org/10.1063/1.531594

View Table of Contents: http://aip.scitation.org/toc/jmp/37/8

Published by the American Institute of Physics

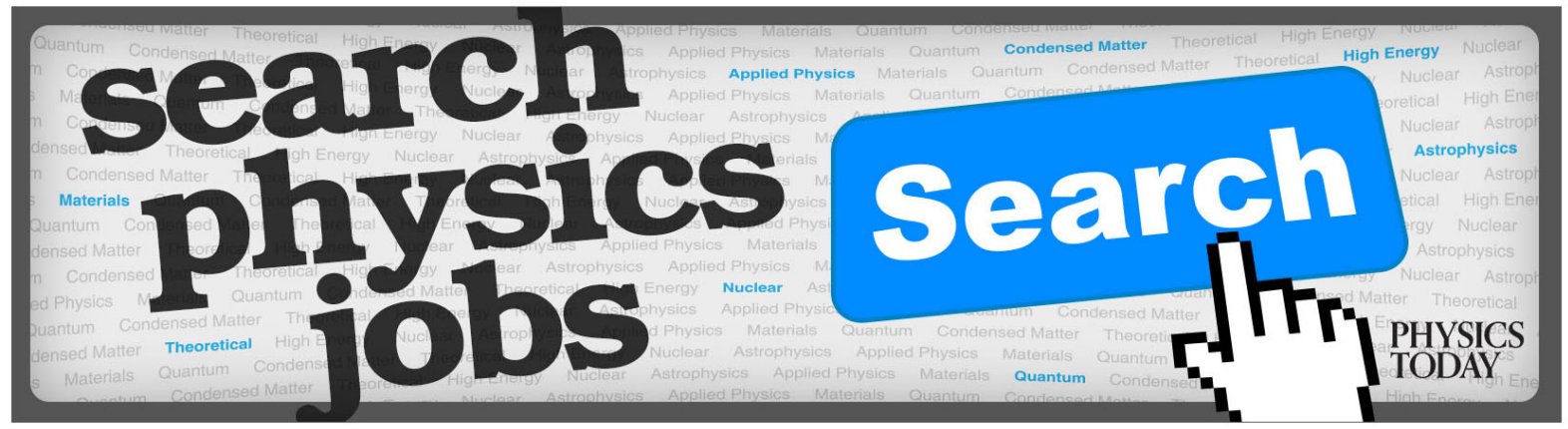




\title{
The $q$-Coulomb problem in configuration space
}

\author{
A. C. Cadavid ${ }^{\text {a) }}$ \\ Department of Physics, California State University, Northridge, California 91330 \\ R. J. Finkelstein ${ }^{\text {b) }}$ \\ Department of Physics and Astronomy, University of California, \\ Los Angeles, California 90095-1547
}

(Received 16 February 1996; accepted for publication 19 April 1996)

We formulate the $q$-Coulomb problem in configuration space with the aid of ladder operators for the radial wave function. The highest angular momentum state corresponding to the principal quantum number $n$ is found to be the monomial $r^{n-1}$ multiplied by a $q$-exponential. The states of lower angular momentum are $q$-associated Laguerre polynomials multiplied by the same $q$-exponential. The state functions all lie in the complex plane and may be interpreted in the standard way. The energy levels are again given by a Balmer formula with $n$ replaced by the basic n. () 1996 American Institute of Physics. [S0022-2488(96)03408-1]

\section{INTRODUCTION}

The $q$-Coulombic problem has been the subject of several studies which differ in the way the Coulombic symmetry is lifted. ${ }^{1,2}$ The basic $\mathrm{O}_{3} \times \mathrm{O}_{3}$ symmetry of this problem may be described in terms of the two vector integrals of the motion, $\mathbf{L}$, the angular momentum integral coming from the spherical symmetry and $\mathbf{A}$, the Lenz vector owing its existence to the $k / r$ potential. The problem separates in spherical coordinates into an angular equation determining the eigenvalues of $\mathbf{L}^{2}$ and $L_{-}$and a radial equation parametrically dependent on $\left(L^{2}\right)^{\prime}$ and determining the eigenvalues of the Hamiltonian $H$ and $\left(\mathbf{A}^{2}\right)$, for which one finds

$$
\begin{gathered}
H^{\prime} \equiv E \sim-\frac{1}{n^{2}}, \\
\left.\left(\mathbf{A}^{2}\right)^{\prime}=m^{2} h^{2}+2 m E\left(\left(L^{2}\right)^{\prime}+\hbar^{2}\right)\right),
\end{gathered}
$$

where $m$ is the mass. Here we discuss the $q$-derived problem by replacing the angular and the radial equations by their $q$-analogs.

\section{THE ANGULAR AND RADIAL EQUATIONS $(q=1)$}

The Hamiltonian for any spherically symmetric potential is

$$
H=\frac{1}{2 m}\left(\frac{1}{r} p_{r}^{2} r+\frac{L^{2}}{r^{2}}\right)+V(r),
$$

where

$$
\left(r, p_{r}\right)=i \hbar \text {. }
$$

The problem separates into an angular equation

\footnotetext{
a'e-mail: acadavid@galileo.csun.edu
}

be-mail finkelstein@physics.ucla.edu 


$$
L^{2} \Phi_{l m}(\theta, \phi)=l(l+1) \hbar^{2} \Phi_{l m}(\theta, \phi)
$$

and a radial equation

$$
\left[\frac{1}{2 m}\left(\frac{1}{r} p_{r}^{2} r\right)+\frac{l(l+1) \hbar^{2}}{r^{2}}+V(r)\right] R(r)=E R(r) .
$$

The solutions of (2.3) are spherical harmonics; and, if $V(r)$ is the Coulomb potential, the solutions of (2.4) are the associated Lagucre functions.

In dimensionless form the radial equation is

$$
R^{\prime \prime}+\frac{2}{x} R^{\prime}+\frac{2}{x} R-\frac{l(l+1)}{x^{2}} R=-\lambda R \text {. }
$$

Set

$$
\rho=x R
$$

then

$$
\rho^{\prime \prime}+\frac{2}{x} \rho-\frac{l(l+1)}{x^{2}} \rho=-\lambda \rho .
$$

The eigenvalue, $\lambda$, as determined from the asymptotic form of this equation, is

$$
\lambda=-\left(\frac{\rho^{\prime \prime}}{\rho}\right)_{x}
$$

We shall discuss the radial equation with the aid of the Infeld ladder method. ${ }^{3,4}$ This procedure sometimes permits the factorization of the Sturm-Liouville operator into the product of a raising and a lowering operator.

Equation (2.7) may be so factored in either of the following ways:

$$
\begin{gathered}
H_{l}^{-} H_{l}^{+} \cdot \rho_{l}^{\lambda}=\left(\lambda+\frac{1}{l^{2}}\right) \rho_{l}^{\lambda}, \\
H_{l+1}^{+} H_{l+1}^{-} \cdot \rho_{l}^{\lambda}=\left(\lambda+\frac{1}{(l+1)^{2}}\right) \rho_{l}^{\lambda},
\end{gathered}
$$

where

$$
H_{l}^{ \pm}=\frac{l}{x}-\frac{1}{l} \pm \frac{d}{d x}
$$

It follows that

$$
\begin{gathered}
H_{l}^{+} H_{l}^{-}\left(H_{l}^{+} \rho_{l}^{\lambda}\right)=\left(\lambda+\frac{1}{l^{2}}\right)\left(H_{l}^{ \pm} \rho_{l}^{\lambda}\right), \\
H_{l+1}^{-} H_{l+1}^{+}\left(H_{l+1}^{-} \rho_{l}^{\lambda}\right)=\left(\lambda+\frac{1}{(l+1)^{2}}\right)\left(H_{l+1}^{-} \rho_{l}^{\lambda}\right) .
\end{gathered}
$$


By comparing (2.8a) with $(2.10 \mathrm{~b})$ and $(2.8 \mathrm{~b})$ with $(2.10 \mathrm{a})$ we see that, up to a normalization,

$$
H_{l+1}^{-} \rho_{l}^{\lambda}=\rho_{l+1}^{\lambda}
$$

and

$$
H_{l}^{+} \rho_{l}^{\lambda}=\rho_{l-1}^{\lambda}
$$

Hence $H_{l+1}^{-}$and $H_{l}^{+}$are raising and lowering operators respectively. There is a highest state given by $\rho \hat{l}$ where $\bar{l}=l_{\max }$ and

$$
H_{\bar{l}+1}^{-} \rho_{\bar{l}}^{\lambda}=0
$$

or

$$
\left(\frac{\bar{l}+1}{x}-\frac{1}{\bar{l}+1}-\frac{d}{d x}\right) \rho \frac{\lambda}{l}=0
$$

The solution of $(2.12)$ is

$$
\rho \frac{\lambda}{l}=x^{\bar{l}+1} e^{-x /(\bar{l}+1)}
$$

When $\rho_{\bar{l}}^{\lambda}$ is substituted in $(2.8 \mathrm{~b})$ we find

$$
\lambda=-\frac{1}{(\bar{l}+1)^{2}},
$$

in agreement with $(2.7)_{x}$.

The energy levels are usually expressed in terms of a principal quantum number $n$ :

$$
\lambda=-\frac{1}{n^{2}},
$$

where

$$
n=\bar{l}+1
$$

Then the radial wave function with maximum $l$ and principal quantum number $n$ is

$$
\rho^{n}=x^{n} e^{-x / n}
$$

or

$$
R_{l}^{n}=x^{n-1} e^{-x / n}
$$

To find states belonging to the same $n$ but with lower values of $l$ one may apply the lowering operator $H_{l}^{+}$. For example we find the three top states (nonrenormalized):
$l=\bar{l}$
$\rho^{n} \sim x^{\bar{l}+1} e^{-x / n}$ 
$l=\bar{l}-1$

$$
\begin{aligned}
& \rho_{\bar{l}-1}^{n} \sim\left[x^{\bar{l}}-\frac{1}{\bar{l}(\bar{l}+1)} x^{\bar{l}+1}\right] e^{-x / n} \\
& l=\bar{l}-2: \quad \rho_{\bar{l}-2}^{n} \sim\left[(2 \bar{l}-1) x^{\bar{l}-1}-\frac{2(2 \bar{l}-1)}{(\bar{l}-1)(\bar{l}+1)} x^{\bar{l}}+\frac{2}{(\bar{l}-1)(\bar{l}+1)^{2}} x^{\bar{l}+1}\right] e^{-x / n},
\end{aligned}
$$

where the quantum numbers of the top state are $(n, \bar{l})$. Expressed in terms of associated Laguerre polynomials, one has

$$
\begin{array}{ll}
l=\bar{l}: & \rho_{\bar{l}}^{n} \sim x^{\bar{l}+1} L_{2 \bar{l}+1}^{2 \bar{l}+1}\left(\frac{2 x}{n}\right) e^{-x / n}, \\
l=\bar{l}-1: & \rho_{\bar{l}-1}^{n} \sim x^{\bar{l}} L_{2 \bar{l}}^{2 \bar{l}-1}\left(\frac{2 x}{n}\right) e^{-x / n}, \\
l=\bar{l}-2: & \rho_{\bar{l}-2}^{n} \sim x^{\bar{l}-1} L_{2 \bar{l}-1}^{2 \bar{l}-3}\left(\frac{2 x}{n}\right) e^{-x / n},
\end{array}
$$

where

$$
L_{n+l}^{2 l+1}(x)=-((n+l) !)^{2} \sum_{\lambda=0}^{n-\lambda-1} \frac{(-)^{\lambda} x^{\lambda}}{\lambda !(n-l-1-\lambda) !(2 l+1+\lambda) !}
$$

\section{THE DEFORMATION}

\section{A. The angular equation}

Replace (2.3) by

$$
L_{q}^{2} \Phi_{l m}^{q}(\theta, \phi)=\langle l\rangle\langle l+1\rangle \Phi_{l m}^{q}(\theta, \phi),
$$

where $L_{q}^{2}$ is the quadratic Casimir of $S U_{q}(2)$ and $\langle l\rangle$ is the basic number:

$$
\langle l\rangle=\frac{q^{l}-1}{q-1} .
$$

Here we have substituted the quadratic Casimir of $S U_{q}(2)$ for the quadratic Casimir of $S U(2)$ and therefore replaced $l(l+1)$ by $\langle l\rangle\langle l+1\rangle$. The eigensolutions of this equation, $\Phi q_{m}$, the $q$-spherical harmonics, have been obtained by Rideau and Winternitz. ${ }^{5}$

\section{B. The radial equation}

We take over (2.2) as a $q$-commutator:

$$
\left(r, p_{r}\right)_{q}=i \hbar
$$

where the conjugate momentum $p_{r}$ becomes the difference operator

$$
p_{r}=\frac{\hbar}{i} D_{r}^{q}
$$

and 


$$
D_{r}^{q} f(r)=\frac{f(q r)-f(r)}{(q-1) r}
$$

We shall abbreviate $D_{r}^{q}$ as $D_{r}$.

There are various possibilities for defining the $q$-radial problem. Here we shall adopt the $q$-modified raising and lowering Eq. (2.11) in dimensionless form, as follows:

$$
\begin{gathered}
H_{\langle l+1\rangle}^{-} \rho_{\langle l\rangle}^{\lambda}=\rho_{\langle l+1\rangle}^{\lambda}, \\
H_{\langle l\rangle}^{+} \rho_{\langle l\rangle}^{\lambda}=\rho_{\langle l-1\rangle}^{\lambda},
\end{gathered}
$$

where we have replaced $l$ by $\langle l\rangle, d / d x$ by $D_{x}$, and therefore $H_{l}^{ \pm}$by

$$
H_{\langle l\rangle}^{ \pm}=\frac{\langle l\rangle}{x}-\frac{1}{\langle l\rangle} \pm D_{x} .
$$

We define the energy by

$$
\lambda=-\left(\frac{D_{x}^{2} \rho}{\rho}\right)_{\infty}
$$

as the natural extension of (2.7).

We take the view that (3.6)-(3.9) describe the full content of the $q$-radial problem, since (3.6), (3.7), and (3.9) imply (2.5) and (2.7) $)_{\infty}$ in the limit $q=1$.

Since these modified equations do not lead to an acceptable Hamiltonian, the energy is fixed not by the eigenvalues of the Hamiltonian, but by the asymptotic form of the wave function via (3.9), or equivalently by poles of the corresponding amplitude in momentum space.

The highest state $(\bar{l})$ is now given by

$$
H_{\langle\bar{l}+1\rangle}^{-} \rho_{\langle l\rangle}^{\lambda}=0
$$

or

$$
\left(\frac{\langle\bar{l}+1\rangle}{x}-\frac{1}{\langle\bar{l}+1\rangle}-D_{x}\right) \rho_{\langle l\rangle}^{\lambda}=0 .
$$

The asymptotic form of this equation is

$$
D_{x} \rho_{\langle\bar{l}\rangle}^{\lambda}=-\frac{1}{\langle\bar{l}+1\rangle} \rho_{\langle\bar{l}\rangle}^{\lambda}
$$

Also

$$
\left(D_{x}\right)^{2} \rho_{\langle\bar{l}\rangle}^{\lambda} \rightarrow \frac{1}{\langle\bar{l}+1\rangle^{2}} \rho_{\langle\bar{l}\rangle}^{\lambda} .
$$

By (3.9) and the preceding equation we have

$$
\lambda=-\frac{1}{\langle\bar{l}+1\rangle^{2}}
$$


or the energy is

$$
E(n, \bar{l})=-\frac{1}{\langle n\rangle^{2}} ; \quad n=\bar{l}+1
$$

The asymptotic form of $\rho_{\langle\bar{l}\rangle}^{\lambda}$ is a solution of (3.12). We see that (3.12) is satisfied by

$$
\rho_{\langle i\rangle}^{\lambda}=\mathscr{E}_{q}\left(\frac{-x}{\langle n\rangle}\right)
$$

where $\mathscr{E}_{q}$ is the $q$-exponential

$$
\mathscr{E}_{q}(y)=\sum \frac{y^{n}}{\langle n\rangle !}
$$

Let the complete solution of $(3.10)$ be

$$
\rho_{\langle\bar{l}\rangle}^{\lambda}=f(x) \mathscr{E}_{q}\left(-\frac{x}{\langle n\rangle}\right)
$$

By the $q$-Leibniz rule

$$
D_{x} P_{\langle n\rangle}^{\lambda}=f(q x)\left(-\frac{1}{\langle n\rangle} \mathscr{E}_{q}\left(-\frac{x}{\langle n\rangle}\right)\right)+\left(D_{x} f(x)\right) \mathscr{E}_{q}\left(-\frac{x}{\langle n\rangle}\right)
$$

Then (3.10) becomes

$$
\left(\frac{\langle n\rangle}{x}-\frac{1}{\langle n\rangle}\right) f(x)=-\frac{1}{\langle n\rangle} f(q x)+D_{x} f(x)
$$

or

$$
\left[(q-1)\langle n\rangle-\frac{(q-1) x}{\langle n\rangle}+1\right] f(x)=\left[1-\frac{(q-1) x}{\langle n\rangle}\right] f(q x) .
$$

If one sets $q=1,(3.20)$ is simply an identity. To discuss this difference equation first convert it to a differential equation by differentiating with respect to $q$ and then setting $q=1$. One finds

$$
\langle n\rangle_{1} f(x)=x f^{\prime}(x)
$$

Therefore

$$
\begin{aligned}
f(x) & =x^{\langle n\rangle_{1}} \\
& =x^{n} .
\end{aligned}
$$

By (3.17) and (3.23) one would have for the highest state,

$$
\rho_{\langle\bar{l}\rangle}^{n}=x^{n} \mathscr{E}_{q}\left(-\frac{x}{\langle n\rangle}\right)
$$


Equation (3.24) has the correct $q=1$ limit but does not satisfy the difference equation (3.20) from which it was derived. Therefore let us return to the difference equation and rewrite it in the following form:

$$
\begin{aligned}
f(x) & =q^{-n}\left(\frac{1+a x}{1+q^{-n} a x}\right) f(q x) ; \quad a=\frac{1-q}{\langle n\rangle}, \\
& =q^{-n} R(x) f(q x),
\end{aligned}
$$

where

$$
R(x)=\frac{1+a x}{1+a q^{-n} x}
$$

By $N-1$ iterations

$$
f(x)=q^{-n N} \prod_{s=0}^{N-1} R\left(q^{s} x\right) f\left(q^{N} x\right)
$$

and

$$
f(x)=\prod_{0}^{\infty} R\left(q^{s} x\right) \lim _{N \rightarrow \infty} q^{-n N} f\left(q^{N} x\right)
$$

Since $|q|<1, q^{-n N} \rightarrow \infty$. Therefore $f\left(q^{N} x\right)$ must vanish in this limit as is also indicated by (3.24). Then (3.28) suggests the ansatz

$$
f(x)=x^{n} \phi(x)
$$

so that by (3.29)

$$
\phi(x)=\prod_{0}^{\infty} R\left(q^{s} x\right) \phi(0),
$$

where

$$
\begin{aligned}
\prod_{0}^{\infty} R\left(q^{s} x\right) & =\prod_{0}^{\infty} \frac{1+a x q^{s}}{1+a x q^{-n+s}} \\
& =\frac{(-a x \mid q)_{\infty}}{\left(-a q^{-n} x \mid q\right)_{\infty}}
\end{aligned}
$$

Here

$$
(x \mid q)_{\infty}=\prod_{0}^{\infty}\left(1-x q^{s}\right)
$$

is the displaced factorial. Set

$$
\phi(0)=1
$$

Then 


$$
f(x)=x^{n} \frac{(-a x \mid q)_{\infty}}{\left(-a q^{-n} x \mid q\right)_{\infty}}
$$

and

$$
\rho_{\langle n\rangle}^{n}(x)=x^{n} \frac{(-a x \mid q)_{\infty}}{\left(-a q^{-n} x \mid q\right)_{\infty}} \mathscr{E}_{q}\left(-\frac{x}{\langle n\rangle}\right)
$$

or

$$
R_{n} \bar{l}(x)=x^{n-1} \frac{(-a x \mid q)_{\infty}}{\left(-a q^{-n} x \mid q\right)_{\infty}} \mathscr{E}_{q}\left(-\frac{x}{\langle n\rangle}\right)
$$

But $^{6}$

$$
(-a x \mid q)_{\infty}=\frac{1}{e_{q}(-a x)}=\frac{1}{e_{q}\left(-(1-q) \frac{x}{\langle n\rangle}\right)}=\frac{1}{\mathscr{E}_{q}\left(-\frac{x}{\langle n\rangle}\right)}
$$

and

$$
\left(-a q^{-n} x \mid q\right)_{\infty}=\frac{1}{\mathscr{E}_{q}\left(-\frac{q^{-n} x}{\langle n\rangle}\right)}
$$

Therefore

$$
R_{n} \bar{l}(x)=x^{n-1} \mathscr{E}_{q}\left(-\frac{q^{-n} r}{\langle n\rangle}\right)
$$

The remaining states are given by (3.7). For example, the three top states are

$$
\begin{gathered}
l=l: \quad \rho_{\bar{l}}^{n} \sim x^{\bar{l}+1} \mathscr{E}_{q}\left(-\frac{q^{-n} x}{\langle n\rangle}\right), \\
l=\bar{l}-1: \quad \rho_{\bar{l}-1}^{n} \sim\left(\rho^{\bar{l}}-\frac{x^{\bar{l}+1}}{\langle\bar{l}\rangle\langle\bar{l}+1\rangle}\right) \mathscr{E}_{q}\left(-\frac{q^{-n} x}{\langle n\rangle}\right), \\
l=\bar{l}-2: \quad \rho_{\bar{l}-2}^{n} \sim\left\{[\langle\bar{l}-1\rangle+\langle\bar{l}\rangle] x^{l-1}-\left[\frac{1}{\langle\bar{l}-1\rangle}+\frac{1}{\langle\bar{l}\rangle}+\frac{\langle\bar{l}-1\rangle}{\langle\bar{l}\rangle\langle\bar{l}+1\rangle}+\frac{q^{-1}}{\langle\bar{l}+1\rangle}\right] x^{\bar{l}}\right. \\
\left.+\frac{1}{\langle\bar{l}\rangle\langle\bar{l}+1\rangle}\left(\frac{1}{\langle\bar{l}-1\rangle}+\frac{1}{\langle\bar{l}+1\rangle}\right) x^{\bar{l}+1}\right\} \mathscr{E}_{q}\left(-\frac{q^{-n} x}{\langle n\rangle}\right) .
\end{gathered}
$$

These are " $q$-Laguerre polynomials" which approach the usual Laguerre polynomials in the limit $q=1$.

The energy levels are given by (3.14). The Coulomb degeneracy is therefore not lifted in this version of the deformation. 


\section{REMARKS}

As already noted, the " $q$-deformation" is not a unique procedure. Reference 1 makes explicit use of the full $O(4)=O(3) \times O(3)$ undeformed symmetry since the integral equation of that paper lies in the $S U(2)$ group space which is carried into itself by the left and right motions (parameter groups) described by $O(3) \times O(3)$. Reference 2 is based on the $q$-deformation of $O(3)$. Since the quantum groups corresponding to $S U(2)$ and $O(3)$ are different, Refs. 1 and 2 describe different deformations. Furthermore, since both solutions lie in their respective algebras, they require a rule for their physical interpretation. In Ref. 7 such a rule is provided in terms of the Hilbert space defined by the algebra itself.

The totally different procedure followed here is based on rules such as the replacement of the radial commutator by the radial $q$-commutator, and further described in Sec. III of this paper. These rules are similar in spirit but formally weaker than the imposition of a quantum group. On the other hand, the wave functions here obtained have the clear advantage of lying in the complex plane as well as being simply related to the $q=1$ solutions from which they are derived. There is therefore no problem of physical interpretation such as we encounter in the other deformations of the Coulomb problem.

It is still possible to make canonical transformations including transformations which exchange the roles of $x$ and $p$ and transform probability amplitudes between configuration and momentum space. For example, the amplitude in $r$-space that we have obtained here may be $q$-Fourier transformed to momentum space, since the momentum operator is a $q$-derivative.

There are in addition quite different canonical transformations, namely

$$
\begin{gathered}
\left(\begin{array}{c}
r \\
p_{r}
\end{array}\right)=T_{q}\left(\begin{array}{c}
R \\
P_{R}
\end{array}\right) \quad T_{q} \in S U_{q}(2), \\
\left(\begin{array}{c}
R \\
P_{R}
\end{array}\right)=T_{q^{-1}}\left(\begin{array}{c}
r \\
p_{r}
\end{array}\right) \quad T_{q^{-1}} \in S U_{q^{-1}}(2),
\end{gathered}
$$

which preserve the $q$-commutator

$$
\left(r, p_{r}\right)_{q}=\left(R, P_{R}\right)_{q}=i \hbar .
$$

In previous work ${ }^{7}$ we have restricted canonical transformations to either $S U_{q}(2)$ or $S U_{q^{-1}}(2)$. Then one can posit that one set of conjugate observables lies in a $q$ - or $q^{-1}$-algebra while the other does not. If the state function turns out to lie in a $q$-algebra, the only invariant that could naturally be associated with a numerical probability of that state would be the Woronowicz integral over the algebra.

'F. L. Chan and R. J. Finkelstein, J. Math. Phys. 35, 3273 (1994).

${ }^{2} \mathrm{~J}$. Feigenbaum and P. G. O. Freund, hep-th 9507116.

'L, Infeld, Phys. Rev. 59, 737 (1941).

${ }^{4}$ E. Schrocdinger, Proc. R. Irish Acad. A 46, 9 (1940).

${ }^{5}$ G. Rideau and P. Winternitz, J. Math. Phys. 34, 6030 (1993).

${ }^{6}$ R. Finkelstein and E. Marcus, J. Math. Phys. 36, 6 (1995).

${ }^{7}$ R. Finkelstein, UCLA/95/TEP/38. 